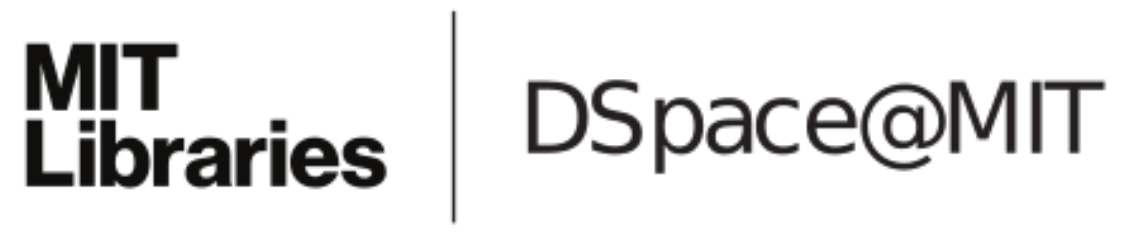

\author{
MIT Open Access Articles
}

Development of CCDs for REXIS on OSIRIS-REX

The MIT Faculty has made this article openly available. Please share how this access benefits you. Your story matters.

Citation: Ryu, Kevin K., Barry E. Burke, Harry R. Clark, Renee D. Lambert, Peter O'Brien, Vyshnavi Suntharalingam, Christopher M. Ward, et al. "Development of CCDs for REXIS on OSIRIS-REx." Edited by Tadayuki Takahashi, Jan-Willem A. den Herder, and Mark Bautz. Space Telescopes and Instrumentation 2014: Ultraviolet to Gamma Ray (July 24, 2014).

As Published: http://dx.doi.org/10.1117/12.2055260

Publisher: SPIE

Persistent URL: http://hdl.handle.net/1721.1/97872

Version: Author's final manuscript: final author's manuscript post peer review, without publisher's formatting or copy editing

Terms of Use: Article is made available in accordance with the publisher's policy and may be subject to US copyright law. Please refer to the publisher's site for terms of use. 


\section{Development of CCDs for REXIS on OSIRIS-REx*}

Kevin K. Ryu , Barry E. Burke ${ }^{\mathrm{a}}$, Harry R. Clark ${ }^{\mathrm{a}}$, Renee D. Lambert ${ }^{\mathrm{a}}$, Peter O’Brien ${ }^{\mathrm{a}}$, Vyshnavi Suntharalingam ${ }^{\mathrm{a}}$, Christopher M. Ward ${ }^{\mathrm{a}}$, Keith Warner ${ }^{\mathrm{a}}$, Mark W. Bautz ${ }^{\mathrm{b}}$, Richard P. Binzel ${ }^{\mathrm{b}}$, Steve E. Kissel $^{\mathrm{b}}$, Rebecca A. Masterson ${ }^{\mathrm{b}}$

a Lincoln Laboratory, Massachusetts Institute of Technology, Lexington, MA 02420

b Massachusetts Institute of Technology, Cambridge, MA 02139

Abstract-The Regolith x-ray Imaging Spectrometer (REXIS) is a coded-aperture soft x-ray imaging instrument on the OSIRIS-REx spacecraft to be launched in 2016. The spacecraft will fly to and orbit the near-Earth asteroid Bennu, while REXIS maps the elemental distribution on the asteroid using $x$-ray fluorescence. The detector consists of a $2 \times 2$ array of backilluminated $1 \mathrm{k} \times 1 \mathrm{k}$ frame transfer CCDs with a flight heritage to Suzaku and Chandra. The back surface has a thin $\mathrm{p}^{+}$-doped layer deposited by molecular-beam epitaxy (MBE) for maximum quantum efficiency and energy resolution at low $x$-ray energies. The CCDs also feature an integrated optical-blocking filter (OBF) to suppress visible and near-infrared light. The OBF is an aluminum film deposited directly on the CCD back surface and is mechanically more robust and less absorptive of $x$-rays than the conventional free-standing aluminum-coated polymer films. The CCDs have charge transfer inefficiencies of less than $10^{-6}$, and dark current of $1 \mathrm{e}^{-} / \mathrm{pixel} / \mathrm{second}$ at the REXIS operating temperature of $-60{ }^{\circ} \mathrm{C}$. The resulting spectral resolution is $115 \mathrm{eV}$ at $2 \mathrm{KeV}$. The extinction ratio of the filter is $\sim 10^{12}$ at $625 \mathrm{~nm}$.

Keywords-Charge coupled device, CCD, x-ray, spectrometer, molecular beam epitaxy, MBE, back-illumination, REXIS, OSIRISREx, optical blocking filter, $\mathrm{OBF}$

\section{INTRODUCTION}

REXIS is a student-built instrument to be included on board the OSIRIS-REx spacecraft. OSIRIS-REx will be launched in 2016 and fly to the near-Earth asteroid (101955) Bennu and bring back samples from its surface in 2023 for further analysis. Bennu is one of a few carbonaceous asteroids that are ideal targets for a sample return mission. These samples will help us understand the origin of life on Earth by providing clues to the composition of organic materials at the beginning of the solar system. REXIS has the objective to map the elemental distribution on the entire surface of the

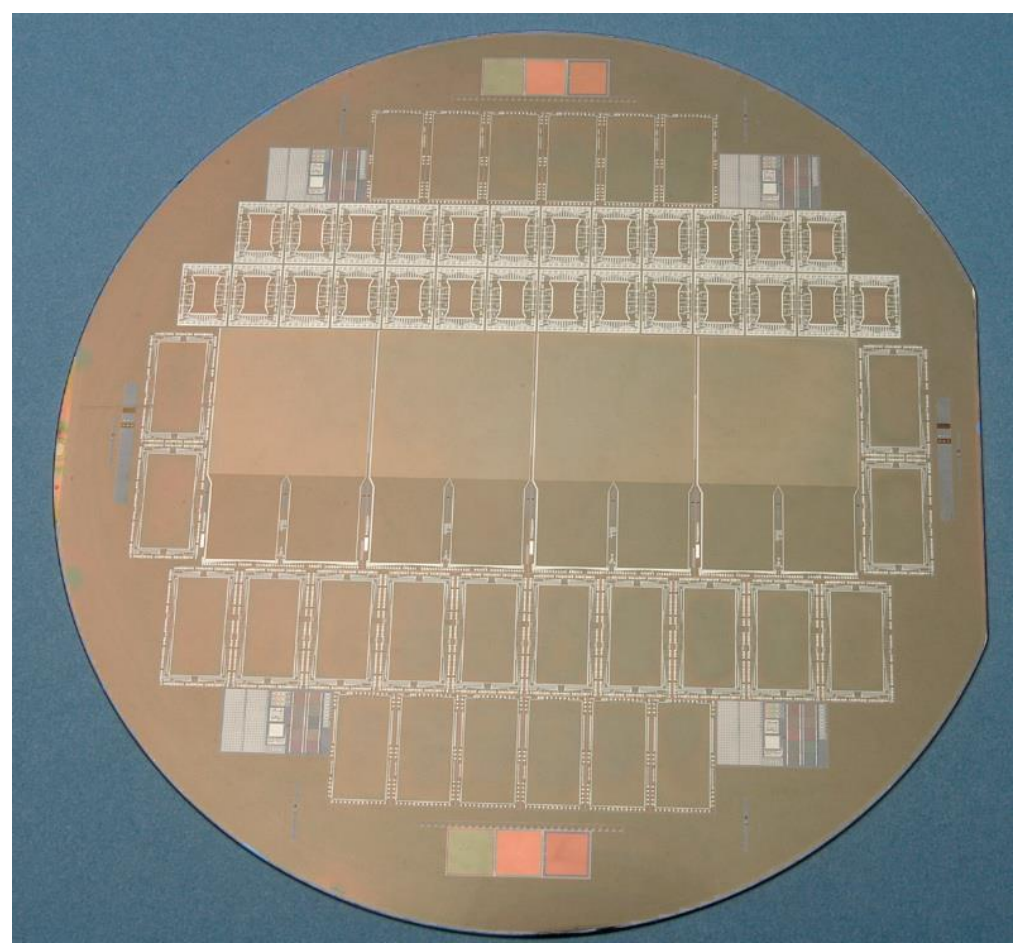

Fig. 1. Photo of a 150-mm wafer with four front-illuminated CCID-41s in the center. asteroid at $50-\mathrm{m}$ resolution using codedmask imaging and $\mathrm{x}$-ray fluorescence (XRF) spectroscopy. The measurements will be used to link Bennu to known meteorite types and enhance the scientific yield of the OSIRIS-REx mission. More information on REXIS is available in [1].

The REXIS focal plane consists of a two-by-two array of MIT Lincoln Laboratory's charge coupled imaging devices (CCID-41s), which are $1 \mathrm{k} \times 1 \mathrm{k}$ frame transfer CCDs with fully depleted silicon thickness of $45 \mu \mathrm{m}$ to enable high quantum efficiency (QE) for 1-10 KeV x-rays. Figure 1 shows a wafer holding four frontilluminated CCID-41s in the center. Twelve such wafers are back illuminated to provide CCDs for the REXIS program. A CCID-41 is a three-layer polysilicon CCD imager with an n-type buried channel and 24- $\mu \mathrm{m}$ image array pixels. The CCDs are fabricated on high-resistivity (>3000 Ohm-cm) floatzone wafers with impurities less than 0.1 ppb that enable deep depletion depth. The CCD imager has a frame-transfer architecture with a $1024(\mathrm{H}) \times 1026(\mathrm{~V})$

Space Telescopes and Instrumentation 2014: Ultraviolet to Gamma Ray, edited by Tadayuki Takahashi, Jan-Willem A. den Herder, Mark Bautz, Proc. of SPIE Vol. 9144, 914440 - (c) 2014 SPIE CCC code: $0277-786 \mathrm{X} / 14 / \$ 18 \cdot$ doi: $10.1117 / 12.2055260$ 


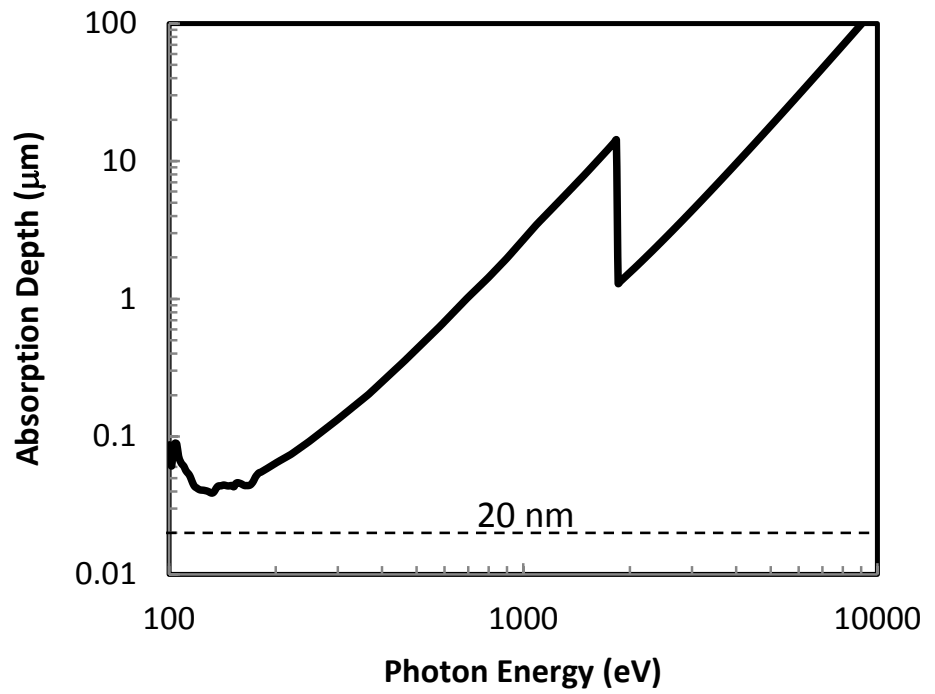

Fig. 2. Photon absorption depth versus energy in silicon. At the absorption depth, approximately $73 \%$ of the X-rays are absorbed. For this reason, it is preferable to have a dead region much less than the absorption depth. The 20-nm thickness of the dead region in REXIS CCD is shown for comparison.

beam epitaxy (MBE) of a silicon layer heavily doped with boron is used. Figure 2 shows the photon absorption depth versus energy in silicon [5]. For soft x-rays, the absorption depth is below $100 \mathrm{~nm}$. A front-illuminated detector configuration will result in loss of most soft $\mathrm{x}$-rays as they must go through oxide and polysilicon gate layers that are thicker than $100 \mathrm{~nm}$. Back illumination with MBE provides smaller dead layers and good charge collection efficiency and results in high $\mathrm{QE}$ and good spectral resolution for soft X-rays.

REXIS utilizes the Sun as the x-ray source obviating the need to carry an x-ray source to illuminate the asteroid. However, the signals generated from visible spectra of the solar radiation will be a noise source for $\mathrm{x}$-ray imaging spectroscopy (XIS) operation. This noise necessitates the use of an optical blocking filter (OBF) that will reduce sunlight by nine orders of magnitude in the visible spectral band. For REXIS, the OBF is integrated directly on the surface of the CCD to enable mechanically robust and high performance filters. Many prior x-ray CCD instruments, including Chandra/ACIS, employ free-standing OBFs, which are mechanically fragile due to their requirement to be thin enough to transmit soft x-rays. This requirement typically drives the thickness to be less than $1 \mu \mathrm{m}$ using a combination of aluminum and polyimide for mechanical support and EUV absorption. Aluminum is a good OBF material due to its high optical density and low atomic number. Table 1 shows thicknesses of materials used for OBF on various previous instruments. To protect free-standing OBF from breaking, precautions were taken in prior programs to minimize potentially damaging acoustic loads during launch, increasing the complexity of the instrument enclosure.

TABLE I. FILTER CHARACTERISTICS FOR CURRENT XIS INSTRUMENTS.

\begin{tabular}{|c|c|c|c|}
\hline \multirow{3}{*}{ Mission/Instrument } & \multicolumn{3}{|c|}{ Blocking Filter Characteristics } \\
\hline & \multirow[b]{2}{*}{ Free-Standing? } & \multicolumn{2}{|c|}{ Composition/Thickness } \\
\hline & & $\begin{array}{l}\text { Aluminum } \\
(\mathrm{nm})\end{array}$ & $\begin{array}{c}\text { Polyimide } \\
\text { (nm) }\end{array}$ \\
\hline Chandra/ACIS & Yes & 130 & 200 \\
\hline XMM-Newton/EPIC ${ }^{\mathrm{a}}$ & Yes & $\begin{array}{l}40 \\
80\end{array}$ & $\begin{array}{l}160 \\
160\end{array}$ \\
\hline Suzaku/XIS & Yes & 120 & 100 \\
\hline Swift/XRT & Yes & 49 & 180 \\
\hline OSIRIS-REx/REXIS & No & 220 & 0 \\
\hline
\end{tabular}

${ }^{\text {a. }}$ EPIC has two filter types. 


\section{BACK ILLUMINATION PROCESS}

All wafer processes described here are done in the Lincoln Laboratory Microelectronics Laboratory (ML), a Class-10 silicon fabrication facility. ML houses all the semiconductor equipment, some developed in-house, required for large-area high-performance CCDs designed at Lincoln Laboratory.

The purpose of the back-illumination process is to enable efficient collection of the photoelectrons generated from $\mathrm{x}$ rays near the back surface. Prior to back illumination, the CCDs are $625 \mu \mathrm{m}$ thick, and the back surface has over $500 \mu \mathrm{m}$ of field-free region. The field-free region causes the electrons to slowly diffuse in random directions before being collected in the pixel capacitor and leads to split events, which degrade spectral resolution. To reduce split events, the silicon must be depleted all the way to the back surface so that the generated electrons are collected by drift. One way to achieve this is to reduce the thickness of the silicon. After the silicon is made thin enough, the back surface must be passivated.

\section{A. Molecular Beam Epitaxy}

The back surface passivation has three purposes. First, it must suppress dark noise generated by the back surface defect states. Second, it must provide a ground plane to terminate fields and remove holes generated from x-rays. Last, it must reflect generated electrons within the silicon. To achieve these three goals, it must have low defect states to silicon, have sufficient conductance, and must repel electrons by having low work function. A heavily boron doped silicon layer meets all three requirements. While meeting the three requirements, this layer should be thin, as generated photoelectrons in this layer are not collected. To make the layer thin while achieving a threshold conductance, high doping density is desired.

While many methods exist to passivate the back surface [6], MBE is an attractive process because of its controllability and ability to make ultra-shallow junctions on the order of a hundred atomic layers. MBE deposits an epitaxial silicon layer at rates of one-tenth atomic layer per second. The extremely slow growth rate is possible owing to the ultrahigh vacuum environment, which lowers the contamination incorporation rate by many orders of magnitude compared to lowpressure chemical vapor deposition. The slow growth rate allows precise thickness controllability down to the atomic layer and high doping density that is not achievable using other film deposition methods at process temperatures allowable for CCDs - above $450{ }^{\circ} \mathrm{C}$, aluminum metallization will alloy with silicon causing device failures [7]. Figure 3 shows doping density of an MBE layer versus that of an ion-implant laser annealed layer. The junction is thinner while the doping density is higher for the MBE process. Due to the higher doping density, higher conductance is achieved with MBE compared to ion-implant laser annealing (IILA), even with a much thinner layer. The MBE passivated devices are

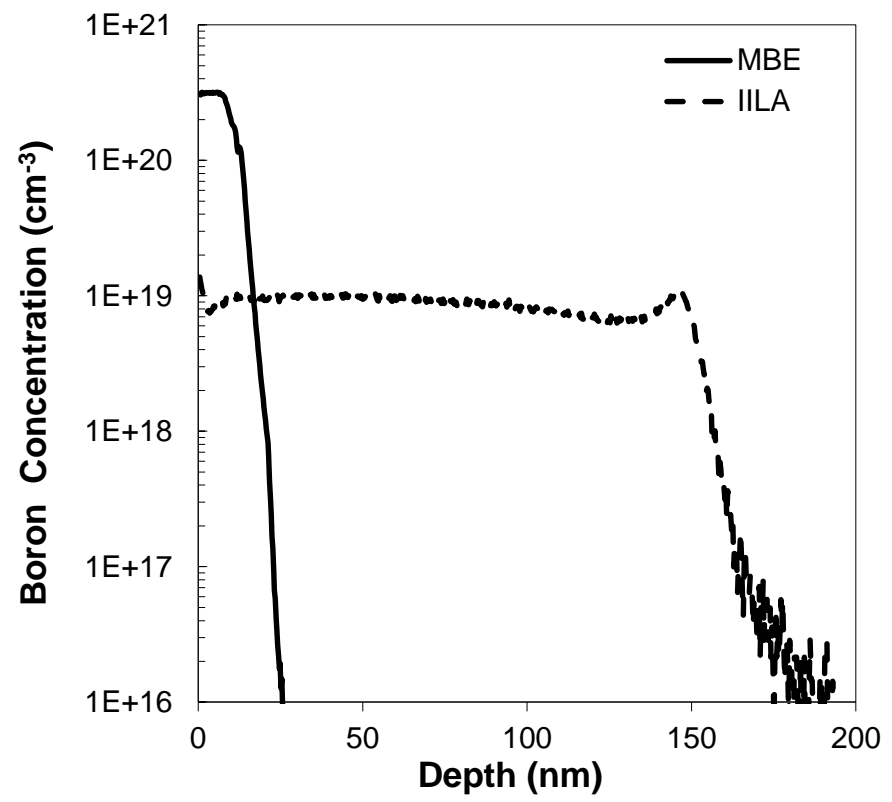

Fig. 3. Doping profile of an MBE deposition. A high doping density over $10^{20} \mathrm{~cm}^{-3}$ is achieved, and an ultra-shallow junction of $20 \mathrm{~nm}$ is formed. Doping profile using ionimplant laser-anneal (IILA) passivation process is shown for comparison. also found to be stable under EUV radiation $[8]$.

Prior to the MBE process, frontilluminated CCD wafers are covered with a low-temperature oxide (LTO) which protects the devices from damage and contamination during the back-illumination process. As mentioned earlier, the field-free regions must be removed. One way of accomplishing this is by rim thinning [7]. This process leaves a rim of 1-mm width intact with full wafer thickness while the center regions beneath the CCDs are thinned to a target thickness of $45 \mu \mathrm{m}$ in a mixture of hydrofluoric acid, sulfuric acid, and acetic acid. The process is engineered to reliably achieve uniformity of $\pm 2 \mu \mathrm{m}$, which allows thinning the device without the need to bond to another carrier wafer. Epoxy bonding cannot be done before the MBE process as the process temperature is typically above $400{ }^{\circ} \mathrm{C}$, and wafers must have minimal outgassing.

To prepare the CCD wafers for MBE, the surface is carefully cleaned in photoresist stripper solvent, and 1:1 hydrogen surface and hydrogen peroxide mixture is used to remove 
organic remnants. Finally, the native oxide is stripped away using 10:1 deionized water and hydrofluoric acid. Care must be taken while handling rim-thinned wafers, as the $45-\mu \mathrm{m}$ center regions are fragile. The rim acts to form a cup to contain the acid while the cleaning is performed. After the native oxide is etched, the wafer is promptly loaded into the MBE machine and the chamber pumped to $10^{-8}$ Torr. Within two hours, it is transferred into the MBE UHV chamber at approximately $10^{-10}$ Torr. MBE is then performed to passivate the back surface with 20 -nm-thick epitaxial silicon that is doped with $2 \times 10^{20} \mathrm{~cm}^{-3}$ boron. After the MBE, an $\mathrm{H}_{2}$ sinter is done to passivate the interface states at the silicon/oxide interface of the CCD. More details of the MBE process can be found in [6] [7].

\section{B. Directly-deposited Integrated Optical Blocking Filter}

After the MBE, wafers are bonded to carrier wafers with epoxy, and silicon is etched to make alignment marks. The OBF must be deposited with a method that does not cause damage to the CCDs. At this point, $\mathrm{H}_{2}$ sinter cannot be done as the wafers are mounted onto carrier wafers with epoxy. Both electron-beam and sputtering deposition of aluminum are damaging to the CCD performance. We have observed that electron beam deposition creates radiation that de-passivates the dangling bonds at the silicon/oxide interface and leads to higher dark current. The energetic nature of the sputtering process is also problematic. For these reasons, thermal evaporation is used to deposit aluminum in high vacuum. Wafers are loaded into the vacuum system and pressure is brought down to $7 \times 10^{-6}$ Torr. The aluminum source in a tungsten boat is heated to aluminum evaporation temperature. Evaporation rate is typically $2 \mathrm{~nm} / \mathrm{sec}$, and $1 \%$ non-uniformity is achieved over a $150-\mathrm{mm}$ wafer, which translates to about $0.1 \%$ of non-uniformity over the surface area of a REXIS CCD. Then 220-nm-thick aluminum is deposited, and the surface is bathed in energized oxygen ions to controllably form 1-nmthick $\mathrm{Al}_{2} \mathrm{O}_{3}$. No degradation in $\mathrm{CCD}$ performance is observed after thermal evaporation of aluminum film on the back surface of the CCD.

One concern with thermal evaporation is that tungsten is also mixed into the film. After evaporation, tungstenaluminum alloying has been observed in the tungsten boat. Energy dispersive X-ray spectroscopy (EDX) has been performed on deposited OBF and no trace of tungsten has been found within measurement limits.

After OBF is deposited, the wafer is patterned and silicon is etched, and pads are exposed to allow electrical probing of the device. An MBE back-illuminated REXIS CCD imager without the OBF coating is shown in Figure 4 (left), and the $\mathrm{CCD}$ imaging single $\mathrm{x}$-ray photons at $5.9 \mathrm{KeV}$ is shown in Figure 4 (right). The charge transfer inefficiency (CTI) is routinely measured to be less than $10^{-6}$.
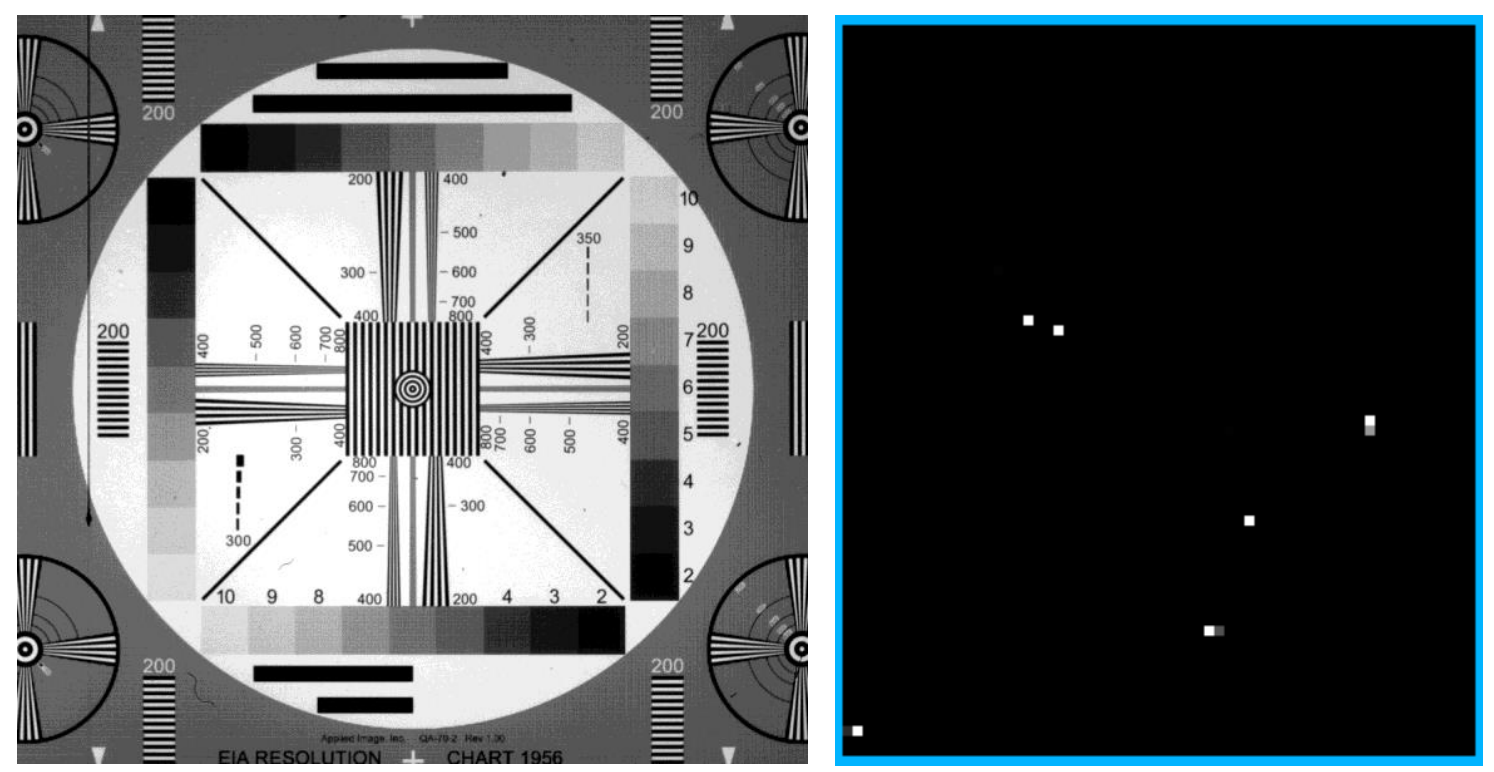

Fig. 4. Image on the left shows resolution target imaging. Excellent cosmetic result is achieved on back-illuminated CCID-41s. Image on the right shows single Fe55 X-ray photons $(\mathrm{E}=5.9 \mathrm{KeV})$ imaged at $-50{ }^{\circ} \mathrm{C}$. From the image, $\mathrm{CTI}$ and responsively is determined. $\mathrm{CTI}$ is less than $10^{-6}$ limited by the measurement accuracy, and responsivity is $19 \mu \mathrm{V} / \mathrm{e}^{-}$. 


\section{CHARACTERIZATION AND DISCUSSION}

The dark current measured over a range of temperatures of a back-illuminated REXIS CCD is shown in Figure 5. At $60{ }^{\circ} \mathrm{C}, 1 \mathrm{e} / \mathrm{pix} / \mathrm{second}$ is achieved. During REXIS operation, the image array will be integrated over a maximum time of four seconds. With a read noise of $2 \mathrm{e}^{-} \mathrm{rms}$, the dark noise will be less than the read noise below $-60{ }^{\circ} \mathrm{C}$. Much lower dark noise is also achievable by using clocking schemes that suppress dark current generation [9].

Energy resolution, $\Delta \mathrm{E}$, is measured in full width at half-maximum (FWHM). Many noise factors contribute to the FWHM - dark noise, CTI, charge collection, read noise, and the Fano noise. Figure 6 shows FWHM measured on a completed MBE back-illuminated device at soft x-ray energies at $-90{ }^{\circ} \mathrm{C}$. The measured FWHM is comfortably below the requirements for REXIS.

The OBF has two competing requirements. It must transmit x-rays while blocking the visible-band radiation. Integrating the OBF directly on the surface of the silicon is advantageous from a handling and performance perspective. The thin film over a large CCD area is challenging to handle and produce. Integrating the OBF on the CCD by depositing the aluminum directly onto the surface of the CCD enables thin OBF without a need for mechanical support material and can increase $\mathrm{X}$-ray transmission while maintaining the same optical blocking qualities.

Prior to this program, it was unclear whether applying aluminum directly on the surface of a high-performance CCD will affect its performance. Although we have observed aluminum on $5 \mathrm{~nm}$-thick MBE film led to degradation in dark current and charge collection efficiency, we found that applying aluminum directly on the back surface of parts with 20 nm-thick MBE does not compromise measurable performance in CTI, dark noise, or the spectral resolution of x-rays.

The x-ray transmission performance of the OBF on a REXIS CCD is shown in Figure 7. On the measured device, a part of the device was not covered with aluminum and was used as a reference. The measured transmission matches expected transmission for 220-nm-thick aluminum based on X-ray absorption lengths published in [5].

The optical performance of the OBF is measured with a 5-mW 625-nm laser beam illuminating the CCD. A Gaussian image of the beam is formed on the imager. By counting the number of electrons generated through the OBF, the optical density (OD) of the OBF is determined to be greater than 12. Based on this measurement, the estimated absorption coefficient of aluminum is $1.3 \times 10^{6} \mathrm{~cm}^{-1}$, which is close to the literature value of $1.5 \times 10^{6} \mathrm{~cm}^{-1}$ at $625 \mathrm{~nm}$ [10]. To characterize the OBF performance over the entire imager, flood illumination is applied to supply approximately $1 \times 10^{15}$ photons $/ \mathrm{cm}^{2}$ - the expected visible flux at REXIS operation. Unexpectedly, about $5 \%$ of the pixels detected light from the flood illumination above the noise floor, as shown in Figure 8. This light leak is thought to be due to pin-holes in the aluminum film that are $100 \mathrm{~nm}$ or less in diameter. Figure 9 shows scanning electron microscope (SEM) images taken on the surface of a REXIS CCD. The texture of the aluminum grain is visible. The images show irregularities in the surface of the aluminum film caused by occasional substrate roughness, and it is plausible that the irregularities produce the pinholes we observe. The size and spatial density of the irregularities are consistent with this interpretation.

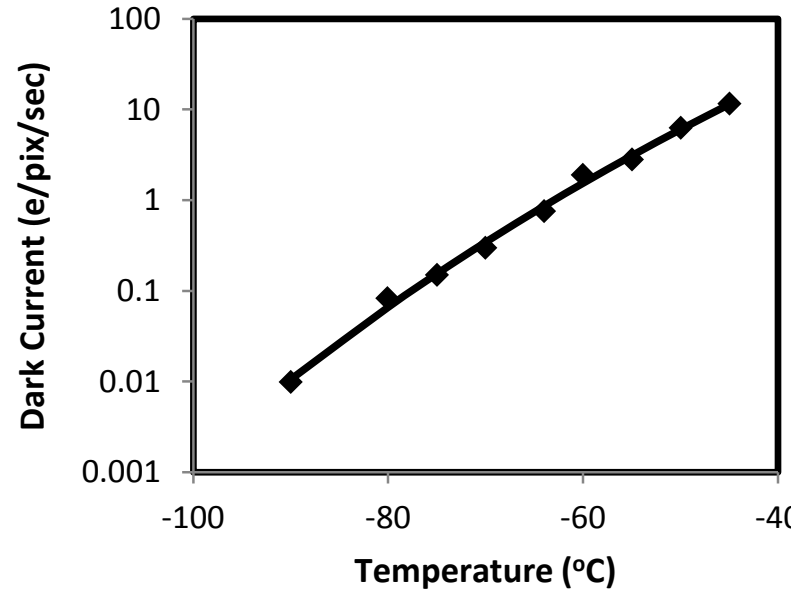

Fig. 5. Dark current versus temperature. Solid line is a fit of the data to an expoential model. Low dark current is achieved due to the good-quality back passivation.

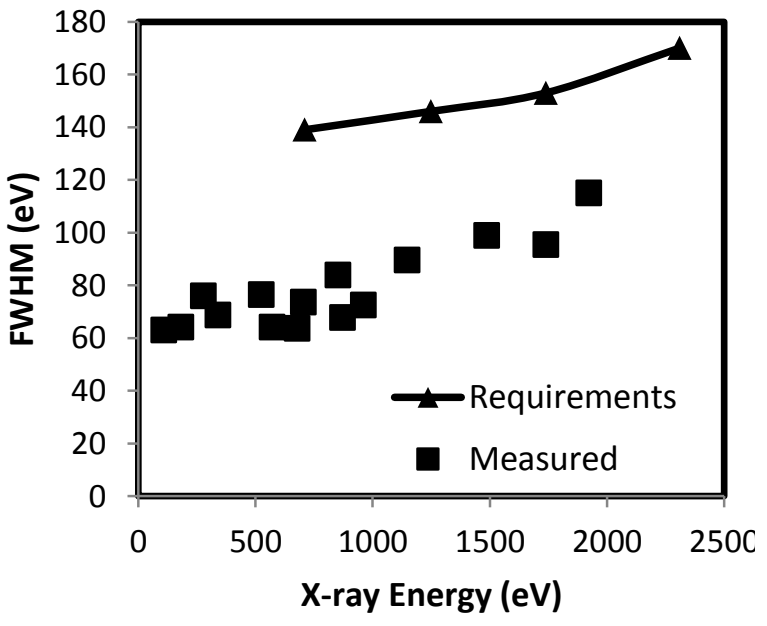

Fig. 6. Spectral resolution measurement of X-ray source at varying energies. FWHM is well below the requirements for the REXIS mission. 


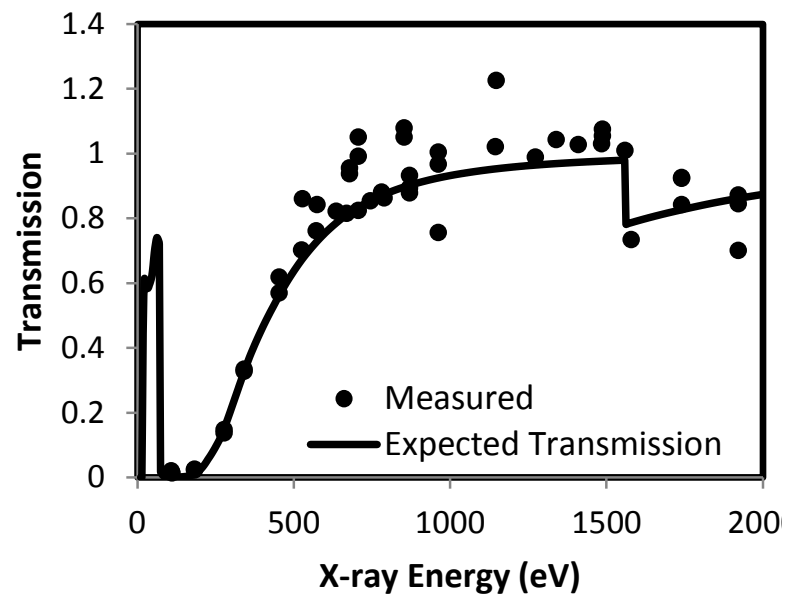

Fig. 7. X-ray performance of the OBF. Solid line is the expected transmission for $220 \mathrm{~nm}$-thick aluminum based on literature values.

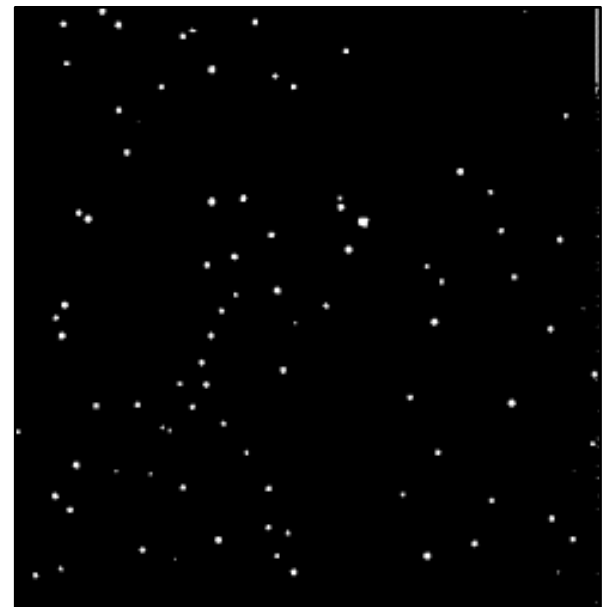

Fig. 8. Large-area optical performance of the OBF. Imager is flood illuminated with uniform light over the CCD and integrated for 20 seconds. Areas where the light is penetrating and visible above the noise floor are shown in white.
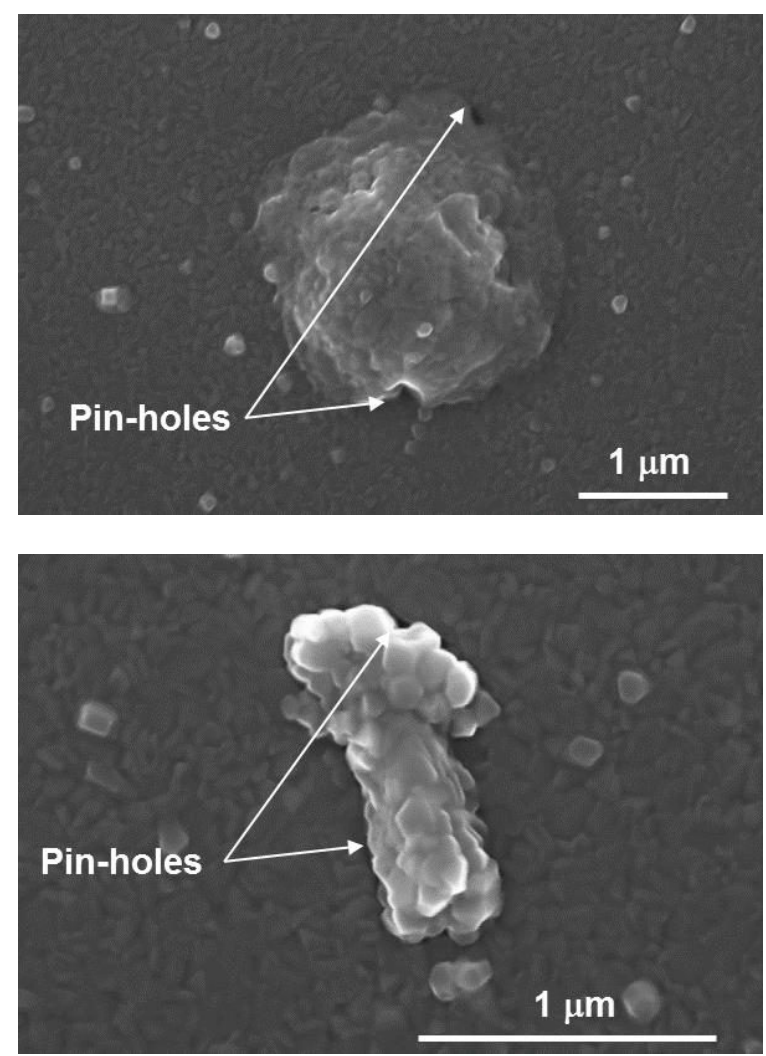

Fig. 9. SEM images of pin-holes in the OBF. Texture of the surface is due to the crystallization of the aluminum film. Pin-holes are smaller than 100 $\mathrm{nm}$ and caused by disruptions on the smoothness of the surface on a submicrometer scale. For perspective, a single image pixel will have an area 50x larger than the field of view of the SEM at this magnification. 

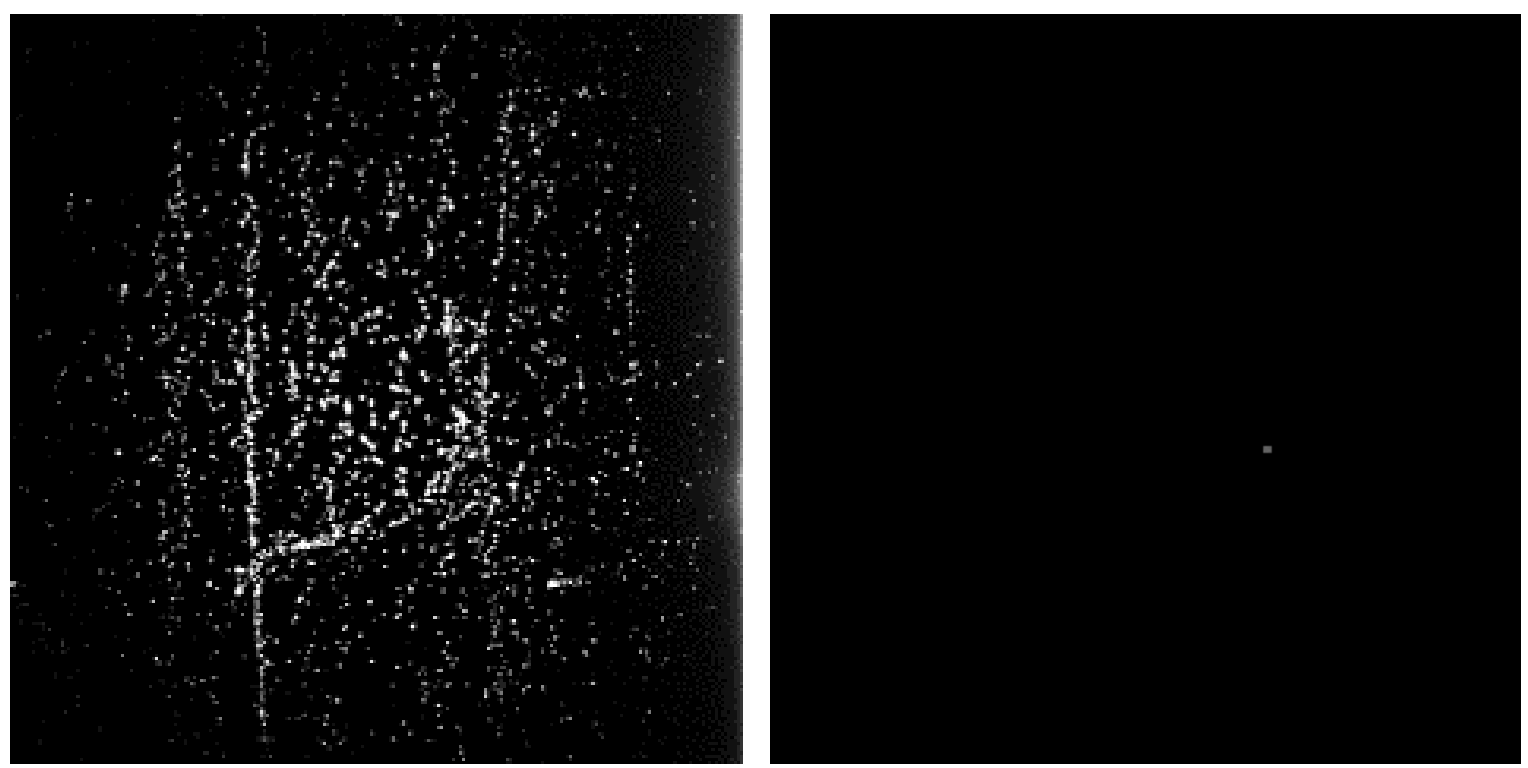

Fig 10. Imager is flood illuminated with uniform light over the CCD and integrated for 20 seconds to characterize optical performance. Areas where the light is penetrating and visible above the noise floor are shown in white. The image on the left is from a device with OBF coating without an interlayer, and the image on the right is from a device with OBF coated over a smoothing interlayer. The interlayer smooths out the surface and suppresses generation of pin-holes in the OBF.

To test whether the surface roughness can explain the pin-holes and transmission of light through the OBF in many locations, a smoothing interlayer consisting of $1 \mu$ n-thick spin-coated photoresist was applied on an experimental device before the deposition of OBF. Figure 10 compares two imagers with and without an interlayer under illumination and shows that the observable number of pixels that detect light through the OBF is greatly reduced with the interlayer. Although the photoresist interlayer is too thick to transmit soft x-rays for REXIS, this result confirms our correlation of the surface roughness of the substrate to the pin-holes and suggests that there are no fundamental limitations preventing fabrication of a pin-hole-free integrated OBF.

\section{SUMMARY}

The back-illumination process and performance of a $1 \mathrm{k} \times 1 \mathrm{k}$ full-frame-transfer CCD for REXIS has been described. MBE is used to achieve an effective 20-nm-thick passivation, and OBF is integrated directly onto the back surface of the CCD to increase mechanical robustness and simplify handling of the filter. The imagers produced have excellent cosmetics, CTI of less than $1 \times 10^{-6}$, and dark current of $1 \mathrm{e}^{\mathrm{e}} / \mathrm{pixel} / \mathrm{second}$ at $-60^{\circ} \mathrm{C}$. The resulting spectral resolution is 115 $\mathrm{eV}$ at $2 \mathrm{KeV}$, and the extinction ratio of the filter is greater than $10^{12}$ at $625 \mathrm{~nm}$. A possible cause of the pin-holes in the OBF has been identified, and it has been demonstrated that a smoothing interlayer reduces pin-holes.

\section{ACKNOWLEDGMENT}

The authors wish to acknowledge the efforts of key personnel in this program. The ML team members all contributed towards fabrication of the CCDs. In particular, the authors thank Doug Young for overseeing the front-illumination process, Donna Lennon for the EDX and SEM analysis, Karen Challberg and Jeffrey Mendenhall for manuscript editing, and Branden Allen at the Harvard-Smithsonian Center for Astrophysics for the helpful discussions. This work was sponsored by the NASA's Strategic Astrophysics Technology Program grant NNX12AF22G to MIT and corresponding IPR NNH12AU04I to Lincoln Laboratory and by NASA contract NNG12FD70C to MIT and corresponding IPR NNG12FC01I to Lincoln Laboratory for REXIS development. The Lincoln Laboratory portion of this work was sponsored under Air Force Contract \#FA8721-05-C-0002. Opinions, interpretations, conclusions and recommendations are those of the author and are not necessarily endorsed by the United States Government. 


\section{REFERENCES}

[1] B. Allen et al., "The REgolith X-Ray Imaging Spectrometer (REXIS) for OSIRIS-REx: identifying regional elemental enrichment on asteroids," in SPIE Optical Engineering + Applications, 2013.

[2] M. W. Bautz, S. E. Kissel, G. Y. Prigozhin, B. LaMarr, B. E. Burke and J. A. Gregory, "Progress in x-ray CCD sensor performance for the Astro-E2 X-ray imaging spectrometer," in High-Energy Detectors in Astronomy, 2004.

[3] K. Koyama et al., "X-Ray Imaging Spectrometer (XIS) on Board Suzaku," Publications of the Astronomical Society of Japan, vol. 59, no. sp1, pp. S23-S33, 2007.

[4] B. Burke, J. A. Gregory, M. Bautz, G. Prigozhin, S. Kissel, B. Kosicki, A. Loomis and D. Young, "Soft-X-ray CCD imagers for AXAF," Electron Devices, IEEE Transactions on, vol. 44, no. 10, pp. 1633-1642, Oct 1997.

[5] B. L. Henke, E. M. Gullikson and J. C. Davis, "X-Ray Interactions: Photoabsorption, Scattering, Transmission, and Reflection at E = 50-30,000 eV, Z =1-92," Atomic data and nuclear data tables, vol. 54, no. 2, pp. 181-342, 1993.

[6] R. Westhoff, B. Burke, H. Clark, A. Loomis, D. Young, J. Gregory and R. Reich, "Low dark current, backilluminated charge coupled devices," in IS\&T/SPIE Electronic Imaging, 2009.

[7] S. Calawa, B. Burke, P. M. Nitishin, A. Loomis, J. A. Gregory and T. Lind, "Substrate preparation and lowtemperature boron doped silicon growth on wafer-scale charge-coupled devices by molecular beam epitaxy," Journal of Vacuum Science Technology B: Microelectronics and Nanometer Structures, vol. 20, no. 3, pp. 11701173, May 2002.

[8] R. C. Westhoff, M. K. Rose, J. A. Gregory, G. D. Berthiaume, J. F. Seely, T. N. Woods and G. Ucker, "Radiationhard, charge-coupled devices for the extreme ultraviolet variability experiment," in Optical Engineering+ Applications, 2007.

[9] B. E. Burke and S. A. Gajar, "Dynamic suppression of interface-state dark current in buried-channel CCDs," Electron Devices, IEEE Transactions on, vol. 38, no. 2, pp. 285-290, 1991.

[10] A. D. Rakic, A. B. Djurivic, J. M. Elazar and M. L. Majewski, "Optical Properties of Metallic Films for VerticalCavity Optoelectronic Devices," Appl. Opt., vol. 37, no. 22, pp. 5271-5283, Aug 1998. 